\title{
Les protéines du choc thermique au carrefour de l'immunologie et de l'endocrinologie
}

Les immunophilines sont des protéines intracellulaires capables de fixer des immunosuppresseurs de la famille de la cyclosporine (CsA) ou du FK 506 et de la rapamycine [1]. Ce sont des peptidyl prolyl cis-trans isomérases ou rotamases, enzymes facilitant le repliement des protéines, et dont l'activité est puissamment inhibée en présence d'immunosuppresseurs. L'inhibition transcriptionnelle de gènes codant pour des lymphokines et en particulier pour l'IL-2, provoquée par la CsA ou le FK 506, fut ainsi interprétée comme le résultat d'un repliement anormal des facteurs transcriptionnels contrôlant l'expression de ces gènes [1]. L'absence d'effet de la rapamycine ou d'analogues de la CsA, molécules ayant toutes un effet inhibiteur de l'activité rotamase, sur la transcription de l'IL-2, vint rapidement remettre en cause cette hypothèse. L'identification de protéines spécifiquement associées aux complexes formés entre les immunophilines et les immunosuppresseurs, permet aujourd'hui d'envisager d'autres mécanismes moléculaires de l'action de ces agents. La calcineurine, une protéine phosphatase activée par la calmoduline en présence de calcium est l'une de ces protéines. Elle fut récemment identifiée comme étant une enzyme clé des voies de transduction du signal émanant du récepteur de la cellule $\mathrm{T}[2,3]$, ce qui explique les conséquences immunologiques que peut avoir l'inhibition de son activité par les immunosuppresseurs. Plus récemment une nouvelle immunophiline appelée p59, appartenant à $m / s n^{\circ} 7$, vol. 8 , septembre 92 la même famille que la FKBP (FK 506 binding protein, protéine fixant le FK 506) a été identifiée par deux approches très différentes. Un groupe américain [4] l'a isolée par chromatographie d'affinité sur une colonne de FK 506. Michel Renoir, dans le laboratoire d'Étienne Émile Beaulieu (Krenulin-Bicêtre), s'intéressait, quant à lui, au complexe protéique du récepteur des stéroïdes au sein duquel il identifia et caractérisa cette protéine [4-6] comme étant une immunophiline liée à une protéine du choc thermique (hsp90), elle-même directement associée au récepteur. L'interaction d'une immunophiline avec une telle protéine chaperon qui se lie aussi bien à des protéines kinases ayant une activité oncogénique qu'à des récepteurs hormonaux ou à des éléments du cytosquelette, pourrait rendre compte de certains des effets directs ou secondaires d'immunosuppresseurs non expliqués par leur inhibition de l'activité phosphatasique de la calcineurine. Les travaux de Michel Renoir montrent aujourd'hui que le complexe immunophiline/protéine du choc thermique/récepteur des stéroïdes, n'est pas dissocié en présence des immunosuppresseurs FK 506 ou rapamycine et que la fixation de ces agents augmente même l'affinité du récepteur pour ses ligands. Outre l'importance fondamentale de ce résultat qui place les protéines du choc thermique au carrefour de l'immunologie et de l'endocrinologie, cet effet pourrait permettre de disposer d'un crible efficace et commode pour trouver de nouvelles molécules à action immunosuppressive.

P.B.

1. Baumann G, Borel JF. Mécanismes moléculaires de l'action des agents immunosuppresseurs. médecine/sciences $1992 ; 8$ : 366-71.

2. O'Keefe SJ, Tamura J, Kincaid RL, et al. FK-506 and CsA-sensitive activation of the interleukin-2 promoter by calcineurin. Nature $1992 ; 357: 692-4$

3. Clipstone NA, Crabtree GR. Identification of calcineurin as a key signalling enzyme in $\mathrm{T}$ lymphocyte activation. Nature 1992 ; 357 : 695-7. 4. Ku Tai PK, Albers MW, Chang $\mathrm{H}$, et al. Association of a 59 kilodalton immunophilin with the glucocorticoid receptor complex. Saince 1992 ; 256: 1315-8.

5. Lebeau MC, Massol N, Herrick J, et al. p59, an hsp 90-binding protein. Cloning and sequencing of its cDNA and preparation of a peptidedirected polyclonal antibody. J Biol Chem 1992 ; 267 : 4281-4.

6. Callebaut I, Renoir JM, Lebeau MC, et al. An immunophilin that binds 6 : Mr 90000 heat shock protein : main structural features of a mammalian p59 protein. Proc Nall Acad Sci USA $1992 ; 89: 6270-4$

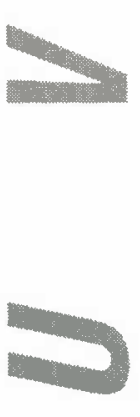

